\title{
The 35-day cycle in the X-ray binary HZ Her/Her X-1
}

\author{
D. Kolesnikov ${ }^{1}$, N. Shakura ${ }^{1,2}$, K. Postnov ${ }^{1,2}$, I. Volkov ${ }^{1,3}$, \\ I. Bikmaev ${ }^{2}$, T. Irsmambetova ${ }^{1}$, R. Staubert ${ }^{4}$, J. Wilms $^{5}$, \\ E. Irtuganov ${ }^{2}$, P. Shurygin ${ }^{2}$, P. Golysheva ${ }^{1}$, S. Shugarov ${ }^{1,6}$, \\ I. Nikolenko ${ }^{3,7}$, E. Trunkovsky ${ }^{1}$, G. Schonherr ${ }^{8}$, A. Schwope $^{8}$ and \\ D. Klochkov ${ }^{4}$ \\ 1 Sternberg Astronomical Institute, Moscow State University, Moscow, Russia \\ ${ }^{2}$ Kazan Federal University, Kazan, Russia \\ ${ }^{3}$ Institute of Astronomy RAS, Moscow, Russia \\ ${ }^{4}$ Institute for Astronomy and Astrophysics, Tubingen, Germany \\ 5 Astronomical Institute of the University of Erlangen-Nuremberg, Bamberg, \\ Germany \\ 6 Astronomical Institute of the Slovak Academy of Scienses, Tatranska \\ Lomnica, Slovakia \\ 7 Crimean Astrophysical Observatory, Nauchny \\ 8 Leibniz Institute for Astrophysics, Potsdam, Germany
}

Received: October 31, 2019; Accepted: February 4, 2020

\begin{abstract}
We present the results of modelling the 35-day superorbital changes in the $B$ and $V$ lightcurves and X-ray flux of HZ Her/Her X-1. The model is implemented in a computer program written in the $\mathrm{C}$ programming language, with a module for parameter optimisation written in Python. The model includes a tilted precessing and warped accretion disc around a freely precessing neutron star. The disc is warped near its inner edge due to interaction with the rotating neutron star magnetosphere. The magnetic torque depends on the precessional phase of the neutron star. The neutron star X-ray emission flux also depends on the free precession phase, which modulates the X-ray illumination of the optical star's atmosphere and the intensity of gas streams. We demonstrate that this model is able to reproduce both the optical observations of HZ Her and the behaviour of the system's 35-day X-ray cycle.
\end{abstract}

Key words: binaries: accretion discs, X-ray - neutron stars

\section{Model}

The donor star shape is defined by an equipotential surface of the Roche potential. The effective temperature of the donor star varies across the surface due to gravity darkening and X-ray irradiation. The disk in the model is warped and tilted. The disk casts a complex X-ray shadow on the surface of the donor star. 


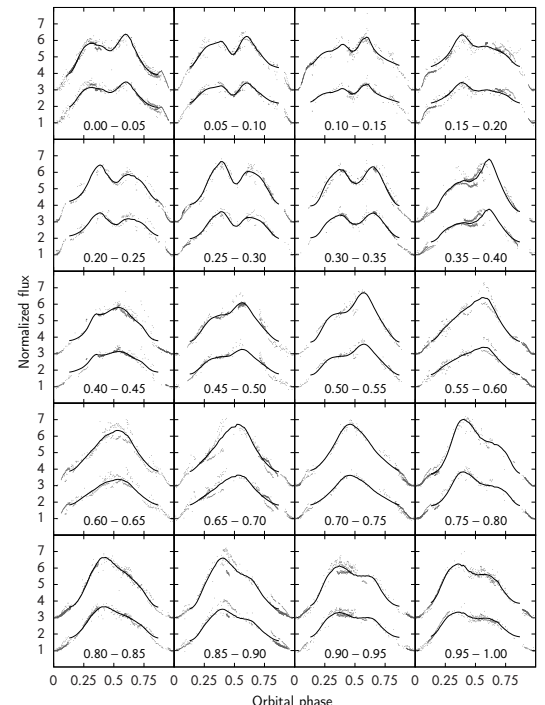

Figure 1. Relative $B$ and $V$ flux, with dots representing the observations and solid lines the theoretical light curves. The numbers in the cells indicate a phase interval in the 35-day cycle. Data in the $B$ band are shifted up 2 units with respect to $V$ data. The optimum theoretical lightcurve for every phase is shown.

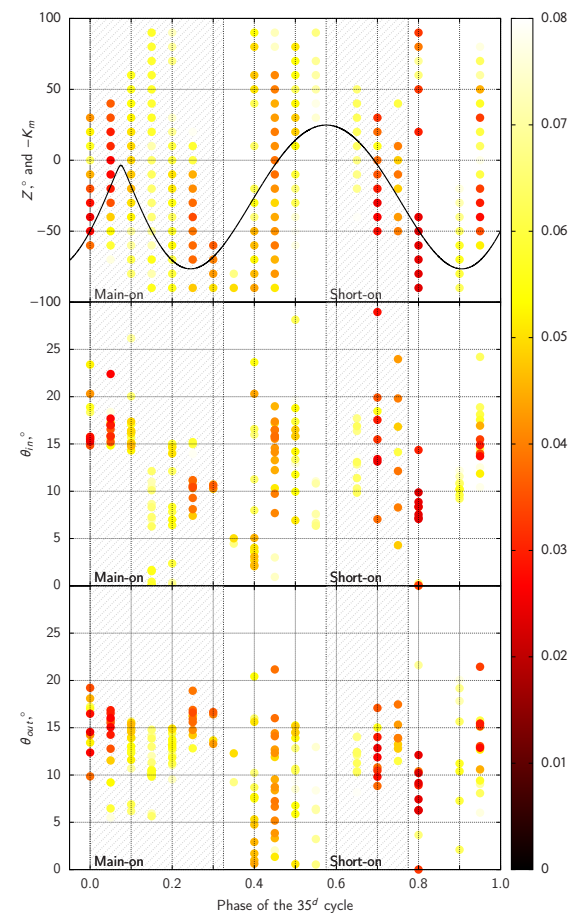

Figure 2. $Z$ is the angle between the nodal lines of the inner and outer edges of the disk. $\theta_{\text {in }}$ and $\theta_{\text {out }}$ are the angles between the disk's inner or outer edge and the orbital plane. The solid line on the first graph is the theoretical magnetic torque $K_{m}$ acting on the disk's inner edge.

We suggest that the disk is warped mostly near the neutron star due to magnetic torque. Near orbital phase 0.5 the disk passes in front of the donor star. We use a ray-tracing technique to determine which parts of the star's surface are obscured by the disk. We have not yet modelled orbital phases near 0.0 , when the disk passes behind the donor star. The neutron star's X-ray intensity is adopted from Postnov et al. (2013). The neutron star is under free precession (Landau et al., 1976) with a period close to 35 days. The code is free and available from https://github.com/eliseys/discostar. 


\section{Results}

Here we show model parameters as a function of phase of the 35-day cycle and light curves corresponding to the minimum of sum of squared residuals between model and observations (Fig. 1). The most interesting result is that the angle $Z$, which is a measure of the torsion of the disk, follows the theoretical magnetic torque $K_{m}$ acting on the disk's inner edge (Fig. 2, Z). The inner edge of the disk tends to be close to the neutron star's equator when the magnetic torque reaches a maximum value (Fig. $2, \theta_{i n}$ ), which was predicted by Lipunov et al. (1981).

\section{References}

Landau, L., Lifshitz, E., Sykes, J., \& Bell, J. 1976, Mechanics, ButterworthHeinemann (Elsevier Science)

Lipunov, V. M., Semenov, E. S., \& Shakura, N. I., Accretion disk orientation in binary X-ray pulsars. 1981, Astron. Zh., 58, 765

Postnov, K., Shakura, N., Staubert, R., et al., Variable neutron star free precession in Hercules X-1 from evolution of RXTE X-ray pulse profiles with phase of the 35-d cycle. 2013, Mon. Not. R. Astron. Soc., 435, 1147, DOI: $10.1093 / \mathrm{mnras} / \mathrm{stt} 1363$ 